\title{
Perspective
}

PERSPECTIVE Actualité en histoire de l'art

1 | 2016

Textiles

\section{Transparence et obstacle : voiles et tissus diaphanes du Moyen Âge en Europe occidentale}

Transparency and Impediment: Diaphanous Veils and Fabrics in the Western European Middle Ages

Francesca Canadé Sautman

\section{CpenEdition}

Journals

Édition électronique

URL : http://journals.openedition.org/perspective/6318

DOI : $10.4000 /$ perspective. 6318

ISSN : 2269-7721

Éditeur

Institut national d'histoire de l'art

Édition imprimée

Date de publication : 30 juin 2016

Pagination : 155-162

ISBN : 978-2-917902-31-8

ISSN : $1777-7852$

\section{Référence électronique}

Francesca Canadé Sautman, «Transparence et obstacle : voiles et tissus diaphanes du Moyen Âge en Europe occidentale », Perspective [En ligne], 1 | 2016, mis en ligne le 15 juin 2017, consulté le 01 octobre 2020. URL : http://journals.openedition.org/perspective/6318 ; DOI : https://doi.org/10.4000/ perspective.6318 


\section{Transparence et obstacle : voiles et tissus diaphanes du Moyen Âge en Europe occidentale}

\author{
Francesca Canadé Sautman
}

Au centre d'un jardin stylisé, une noble dame et un homme arborant une couronne jouent aux échecs. Coup de vent ou émoi soudain ? Les doubles pans du voile de la dame s'envolent, légers, diaphanes, empiétant sur les bords du cadre. Leur débordement sur le cadre de la scène révèle l'ornementation du tissu sous les plis superposés - convention picturale du transparent (fig. 1). L'auteur de cette enluminure du Psautier de Luttrell, composé en Angleterre entre 1325 et 1340, apporte un soin méticuleux à produire l'effet de transparence ${ }^{1}$. Les arts visuels du XIV siècle voient déjà la multiplication de cet effet : outre ces légers tissus ornant la tête des femmes dans les marginalia aux sujets profanes, la présence des textiles transparents se fait sentir dans la peinture religieuse. $\mathrm{Au} \mathrm{XV}^{\mathrm{e}}$ siècle, les tissus diaphanes vont occuper bien plus visiblement encore l'espace pictural profane autant que religieux, déployant un grand faste de nuances de luminosité. Néanmoins, les références textuelles à de tels tissus et à leurs usages restent des mentions succinctes qui négligent leurs effets de transparence alors même que de nombreux types de textes attestent de la production et de l'échange de divers tissus de laine et de soie en Europe au cours du Moyen Âge. Une telle disproportion suggèrerait que ces tissus, même faits de soie ou de lin de qualité, étaient peu valorisés comme objets matériels, contrairement à leur importance comme motifs picturaux. C'est d'abord cet écart entre les données textuelles et visuelles qui nous mène à interroger ces tissus largement délaissés dans l'imposant corpus critique et historique sur la production textile médiévale ${ }^{2}$.

Les tissus de lin, de soie ou de coton, ou composés d'un mélange de ces matériaux sur chaîne et trame, légers mais opaques, mollement pliés ou en épais drapés, ont fait partie du vêtement de tête féminin au moins depuis le $\mathrm{XI}^{\mathrm{e}}$ siècle, devenant à la fois plus transparents, plus variés et plus ornementaux dans les témoignages picturaux des coiffures compliquées des XIV et $\mathrm{XV}^{\mathrm{e}}$ siècles ${ }^{3}$. Or, dès le XIV ${ }^{\mathrm{e}}$ siècle, le tissu diaphane dans les représentations de la Crucifixion relève d'une symbolique où matérialité et spiritualité sont imbriquées. La chronologie des données matérielles, lexicales et picturales concernant ces tissus s'avère donc non-linéaire et ce sont leurs recoupements qui permettent de reconstituer une vie médiévale du tissu diaphane.

\section{Les tissus transparents comme objets}

Nonobstant la relative pénurie de sources textuelles qui identifient explicitement la catégorie du transparent, de tels textiles ont bel et bien existé et depuis longtemps. Un textile non-tissé, la résille ou filet, était déjà fabriqué à l'époque néolithique par la technique de torsion dite sprang ${ }^{4}$, produisant un artefact de fils de soie retrouvé relativement fréquemment lors de fouilles archéologiques. Il figure dans l'habillement de tête féminin jusqu'à la fin du Moyen Âge et il est connu sous le nom de " crespine " ou " crespinette " (caul en anglais), à la lisière des XIII et XIV ${ }^{\mathrm{e}}$ siècles, comme composante de savantes coiffures réticulaires. Les artisans " crespiniers " étaient réglementés en corporation produisant des résilles de fil de soie pour l'habillement mais

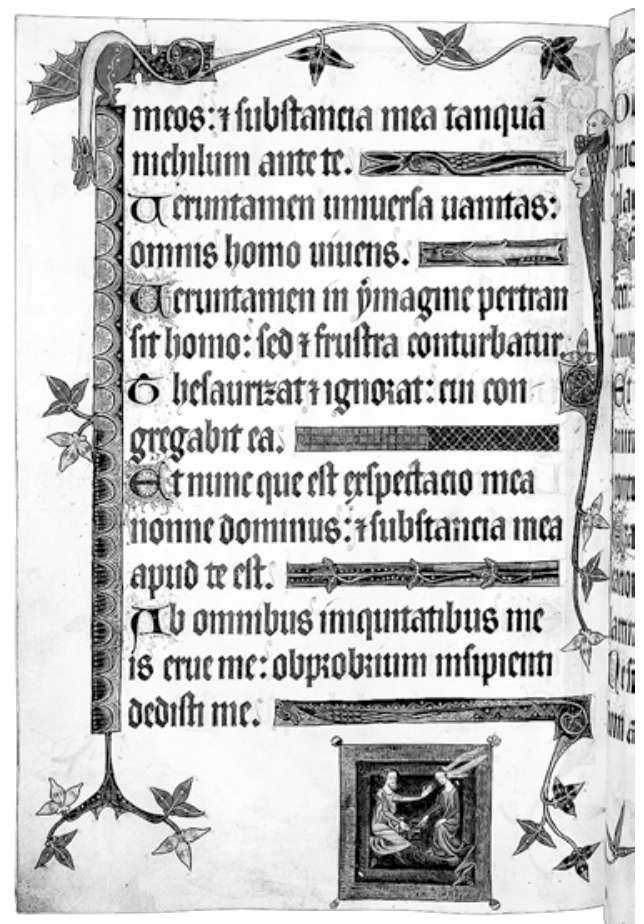

1. Psautier de Luttrell, 1325-1340,

Londres,

British Library, Add MS 42130, fol. $76 \mathrm{v}^{\circ}$. 
aussi pour les ornements de coussins et les devants d'autels 5 . Par ailleurs, les Vikings importaient massivement la soie venant du MoyenOrient et des pourtours de la Méditerranée et de nombreux fragments de soie, dont certains proches de la gaze, ont été recueillis dans des sites britanniques marqués par leur influence, à York et à Dublin ${ }^{6}$. Des restes de tissu de crêpe ont été également retrouvés dans les pages de garde d'une Bible de Théodulfe, évêque carolingien ${ }^{7}$.

Le crêpe et la gaze, de soie ou de lin, sont les tissus légers les plus souvent mentionnés dans les sources médiévales relatives au commerce et aux usages. Le crêpe, tissé sans " croisure, comme les étamines, sur le métier à deux marches ", à l'origine importé d'Orient, devient aux XII et XIII ${ }^{\mathrm{e}}$ siècles un monopole de Bologne, jusqu'à ce que le roi de France Henri IV fonde des manufactures au château de Mantes en 1604. Tissu ajouré, tissé avec des fils écartés, la gaze tire son nom de la ville de Gaza ; sa fabrication suivait une technique amplement appliquée au lin dans l'Europe médiévale et qui avait été développée en Chine pour le tissage de la soie sous la dynastie des Han ${ }^{9}$. D'abord importées ${ }^{10}$, ces gazes de soie se multiplient en Europe avec l'implantation, à partir du XII ${ }^{\mathrm{e}}$ siècle, des ouvroirs à soie en Sicile et avec l'essor de l'industrie de la soie dans les villes italiennes de la fin du Moyen Âge. Pourtant, gazes et crêpes de soie ne sont mentionnées ni dans les descriptions émerveillées des ateliers dont Roger de Sicile est le commanditaire, ni parmi les typologies de soies produites à Lucques ou à Florence au Trecento ${ }^{11}$. Cependant, elles apparaissent dans des interdits somptuaires ${ }^{12}$ dont l'objet est de limiter les velléités au luxe : le roi Edouard IV d'Angleterre (1442-1483) interdit la vente de couvre-chefs, de guimpes, de voiles, et de linon [lawn] au-dessus de 10 shillings ${ }^{13}$.

\section{Des objets au lexique}

Le champ lexical des tissus transparents médiévaux suit avant tout une optique très différente de celle du monde moderne. Les dictionnaires archéologiques offrent quelques points de départ mais ils restent limités et varient peu entre eux. Ainsi, Camille Enlart identifie trois types de tissus légers de lin et de soie, dont le bouquerans ou bougran, probablement une forme dérivée du byssus antique, avant que son imitation en Europe au XIV ${ }^{\mathrm{e}}$ siècle n'en fasse une toile grossière ; les couvrechefs $\mathrm{s}^{14}$ de soie ou de lin ; et le crêpe de soie, de laine ou de $\operatorname{coton}^{15}$. Cette nomenclature réduite suggère que les notions de transparence et d'opacité ne répondent pas à la façon dont les tissus étaient désignés au Moyen Âge ; Kurt Zangger identifiait plutôt les critères de combinaison du dessin et de la couleur, de provenance, de coloris et enfin, de qualité et de prix ${ }^{16}$.

Il nous faudra donc retrouver cette typologie à travers une combinaison d'autres indices lexicaux : les noms de fibres et tissus de base (soie, chainsil [lin]), certains articles vestimentaires, chainse, chemise, couvrechef, guimpe, voile, des gestes, tels que " lier " un tissu léger en multiples plis noués, et un adjectif, "délié17 ". Ainsi des textes de romans évoquent le " chainsil délié " dont les effets de transparence sont parfois malencontreux, quand il remplace une armure par exemple $^{18}$. La chemise, portée contre le corps, pouvait être faite du lin le plus fin - «plus délie d'un fil d'iragne ${ }^{19} "$ - ou le plus grossier, voire même de soie, sans pour autant être vraiment transparente, mais les textes littéraires soulignent qu'elle laisse deviner le corps et ses zones les plus interdites ${ }^{20}$. La chemise médiévale se situe alors au bord d'une transparence suspecte et, lorsqu'elle est le seul vêtement porté, elle devient une forme de nudité et signale que le sujet risque d'être bientôt coupé du tissu social ${ }^{21}$.

$\mathrm{Au} \mathrm{XV}$ siècle, si le costume a changé, l'adjectif " délié " continue à codifier la transparence des tissus utilisés. C'est ainsi que le chroniqueur Monstrelet vers 1467 parle de « deliez couvrechiesfs par-dessus qui pendoient par derriere jusqu'à terre $^{22}$ ". Le voile " délié " placé sur le visage de Jeanne de Bourbon lors de ses funérailles est si transparent "Que tout plainement on voioit son visage parmy ${ }^{23}$." Le lin acquiert parfois une finesse quasiment transparente, comme on peut le lire dans un passage du Trésor de la cité des dames... (ou Le livre des trois vertus) de Christine de Pisan (1364 - vers 1430) critiquant une femme de statut non-noble dont la dépense luxueuse pour un somptueux appareil de gésine comprend un drap de " lin délié " si fin qu'on le prenait pour de la soie.

Du simple matériau on tend à passer à la notion, culturelle et sociale, de voile, et à l'appliquer à tous ces tissus pendants et enveloppants, par extension du vocabulaire usité dans l'histoire du costume. Le voile tel qu'il est connu aux XII et XIII ${ }^{\mathrm{e}}$ siècles est en fait sombre et possède bien la qualité de cacher, de céler, par exemple, l'identité d'un protagoniste ${ }^{24}$ 


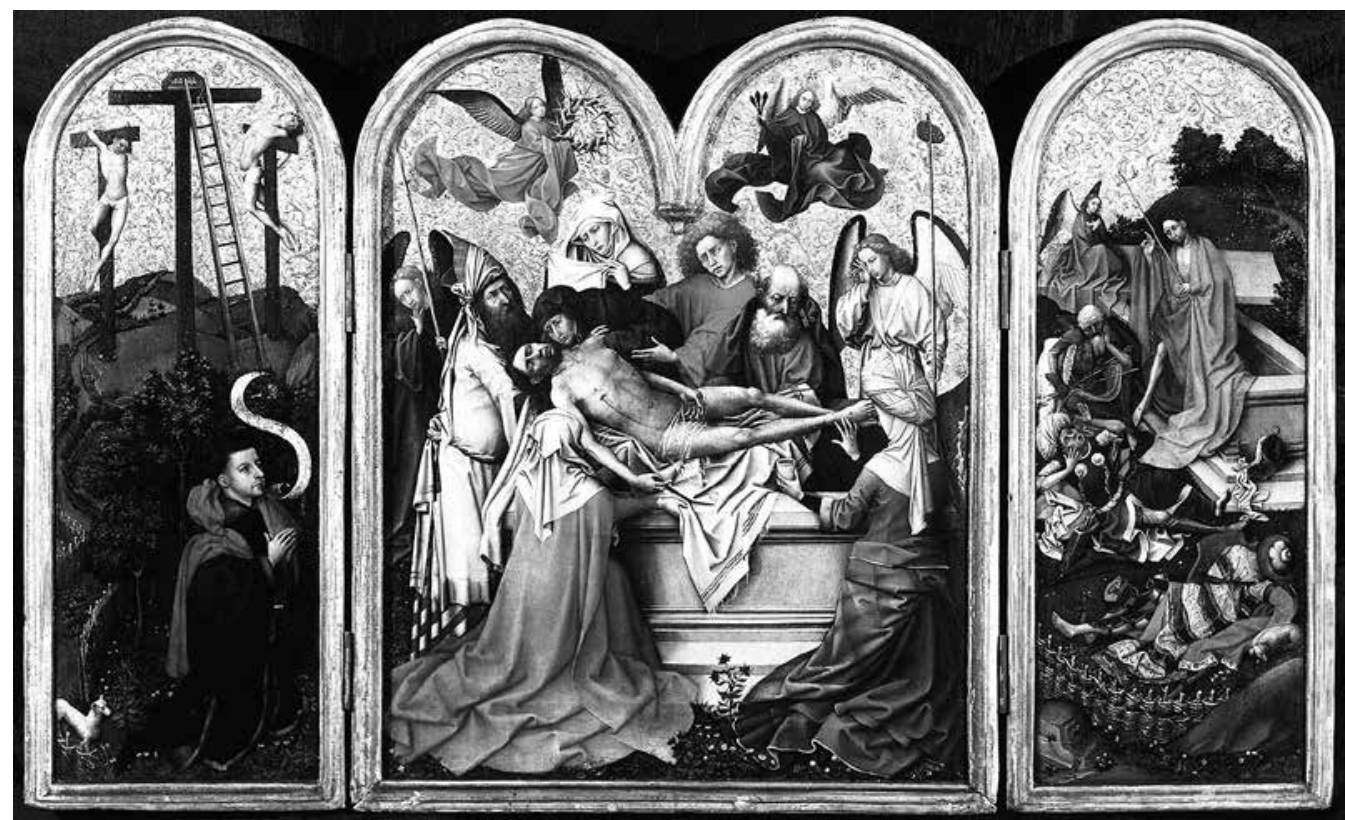

2. Robert Campin, Descente au tombeau du Triptyque de Seilern, vers 1410 , Londres,

The Courtauld Institute of Art.

\section{Voiler le corps sacré}

Alors que le pagne ou la tunique portés par le Crucifié étaient figurés par des couleurs mates et sombres auparavant, la peinture du XIV siècle commence à opter pour une représentation du périzonium sous la forme d'un linge blanc, parfois en plis volumineux cachant effectivement le corps, parfois rendu si transparent qu'on en devine les contours, tendance initiée semble-t-il par Giotto vers 1304-1306. Dès ce moment, la transparence sera souvent mobilisée dans la représentation du mystère christique ${ }^{25}$. La Descente au tombeau peinte par Robert Campin vers 1410 dans le Triptyque de Seilern, conservé à l'Institut Courtauld de Londres (fig. 2), est exemplaire de ce " voile " apposé par peintres et enlumineurs sur le corps du Christ, qui semblent vouloir révéler l'humanité du Christ dans la pose d'un corps dénudé tout en couvrant ses parties génitales (quoique certains aient signalé une vague présence anatomique sous le linge) - écran symbolique contre le regard profane. Dans une Crucifixion plus tardive (1440), Rogier van der Weyden ${ }^{26}$ a osé quant à lui dévoiler toute l'anatomie du Christ à travers l'envol du pagne éthéré. Jean-Claude Schmitt et Jérôme Baschet ont interprété ce problème d'un Christ sexué ou asexué sous le voile en relisant la présence ou l'absence de parties corporelles comme l'émanation d'une nature divine inclassable suivant une corporalité humaine, s'opposant à la fois à la théorie naturaliste et à celle de Leo Steinberg centrée sur le concept " d'humanation ${ }^{27}$ ". Un autre éclairage est possible, selon la tradition théologique, émergeant $\mathrm{au} \mathrm{XI}^{\mathrm{e}}$ siècle, qui fait de la chair même du Christ un rideau mystique et associe son incarnation à un voile de forme humaine descendant sur sa nature divine ${ }^{28}$.

Le voile presque imperceptible porté par la Vierge dans la peinture des Trecento et Quattrocento italiens est une autre forme du voile-écran, marque de divinité qui a précédé les ornements de tête diaphanes que l'on trouve dans la peinture du $\mathrm{XV}^{\mathrm{e}}$ siècle. La Vierge et certaines saintes portent en effet un premier voile de tête transparent posé directement sur leurs cheveux, souvent recouvert à demi par un ou plusieurs pans de tissus opaques. Ce voile qui ne cache rien et en nécessite un deuxième, conformément aux normes de l'habillement féminin, n'est donc pas seulement d'ordre vestimentaire, évoquant la "pureté ", mais suggère aussi la comparaison souvent faite au Moyen Âge de la Vierge avec un verre infrangible captant la lumière.

Cependant ce voile n'apparaît pas toujours tissé dans un matériau à peine visible : la Vierge porte à même ses cheveux un voile de gaze transparente tissée de bandeaux horizontaux aux motifs étoilés dans un tableau de l'atelier de Botticelli $^{29}$ (vers 1490), et un tissu à la facture 


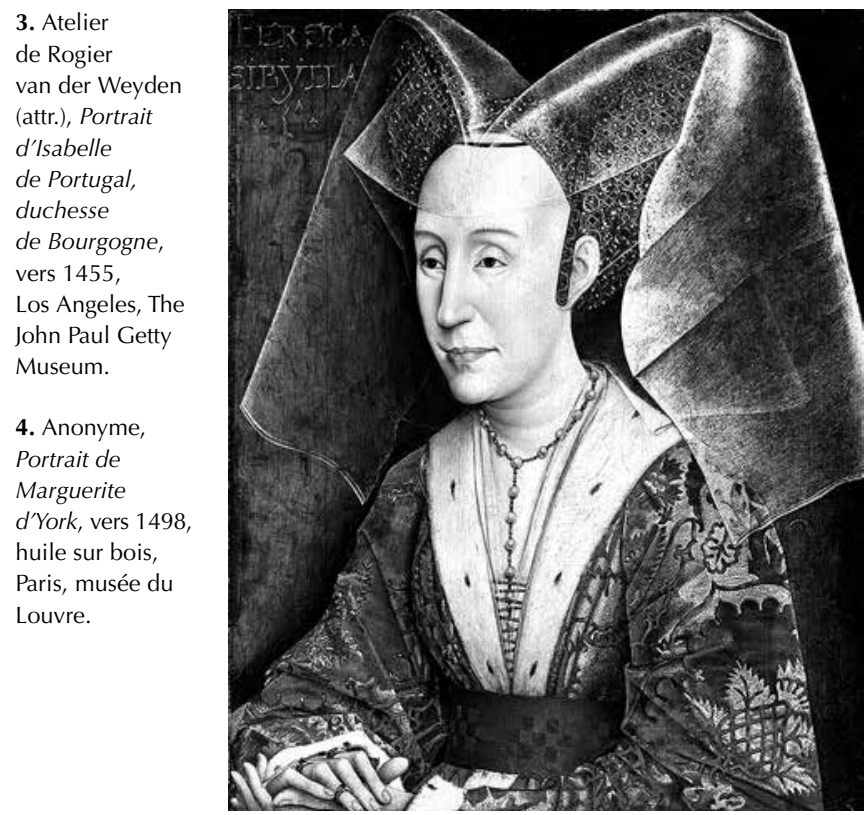

complexe très similaire (transparent mais marqué de stries horizontales) figure dans un manuscrit français de la même époque ${ }^{30}$.

\section{Nouvelles techniques et voiles lumineux}

Ce voile, ou plutôt ce voilage, qui semble au premier abord " inutile ", refait surface dans les coiffes féminines aux XV ${ }^{e}$ et XVI $I^{e}$ siècles dans les tableaux et manuscrits ${ }^{31}$. Il ne s'agit plus alors du voile qui pend à l'arrière d'une coiffure ou d'un couvre-chef, hennin ou autre - la transparence arborée par les femmes est un simulacre d'écran, morceau de gaze limpide et transparent tiré sur le front ou encadrant le visage, constituant un fragile obstacle au regard. Les effets de transparence totale déjà atteints par les peintres italiens au XIV siècle, grâce à la technique de la tempera sur bois, vont être haussés à de nouvelles nuances et à des jeux de lumière contre l'étoffe savamment étudiés avec l'essor de la peinture a l'huile au milieu du XVe siècle. Quoique celleci n'élimine pas la peinture à la tempera qui continuera de cerner la transparence jusqu'à la fin du Xve siècle, elle permettra d'innombrables variations dans la figuration des tissus complètement perméables à la lumière ou partiellement opaques - en Flandres et en Bourgogne comme en Italie, aux alentours de $1430^{32}$. Dans l'habillement féminin, tout en se faisant de plus en plus éthéré, le tissu léger devient un élément

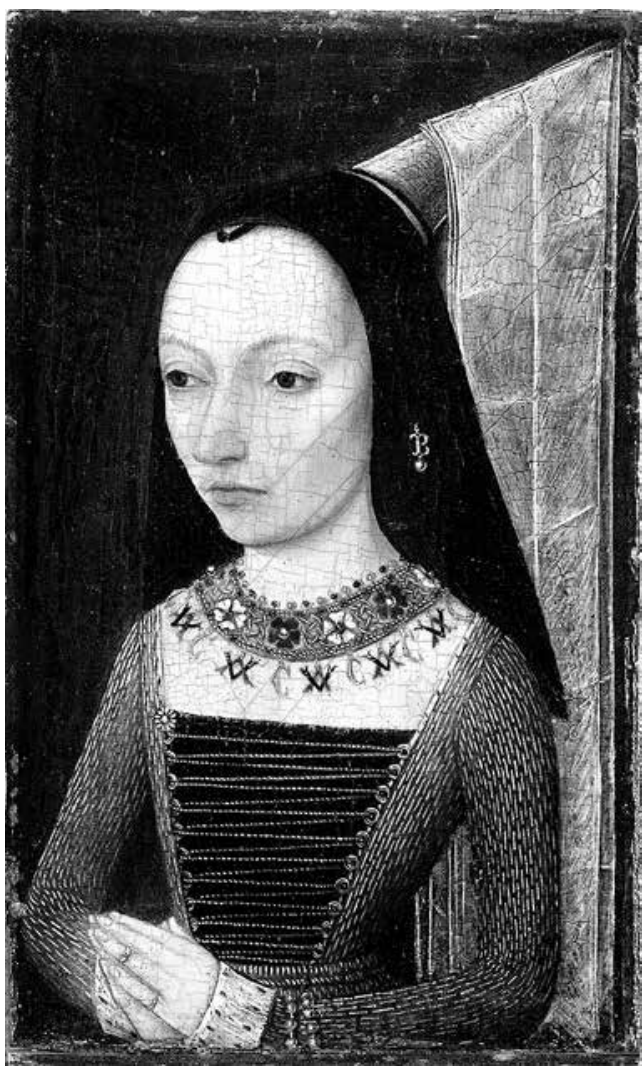

indispensable de la construction de coiffures recherchées, très éloignées des principes de piété et de modestie. Toute une gamme de tissus diaphanes semblent alors composer l'habillement de tête, des turbans orientalistes de la Madeleine et des femmes bibliques, en passant par la pudique couverture du buste dans les tableaux des $\mathrm{XV}^{\mathrm{e}}$ et XVI $\mathrm{XI}^{\mathrm{e}}$ siècles, sans oublier la barbette de deuil des veuves de souche royale ou impériale. Marguerite d'Autriche, régente des Pays-Bas pour son neveu Charles-Quint, la porte dans tous ses portraits officiels ${ }^{33}$.

De nombreux exemples montrent comment la peinture septentrionale, forte de ces nouvelles techniques, capte les nuances entre transparence absolue et opacité dans la représentation de tissus et voilages. Dans un portrait d'Isabelle de Portugal, épouse de Philippe le Bon, attribué à l'atelier de Rogier van der Weyden (vers 1455 ; fig. 3), la duchesse porte une somptueuse robe de brocart rouge $^{34}$, ses cheveux sont tirés et cachés sous la coiffe, son front épilé. Elle arbore une double cornette argentée émaillée de pierres précieuses et, par-dessus, un voile qui pend jusqu'aux épaules 
et se déploie sur le front. Ce voile, le peintre l'a rendu à la fois léger, doux, élastique, et translucide. Il brille d'un éclat argenté telle une gaze de soie à la fois moelleuse et transparente. Dans une œuvre attribuée à Hans Memling considérée comme allégorique, La Dame à l'xillet (vers 1485-1490), le peintre semble démontrer toute sa maîtrise dans le passage graduel, le long du voile d'atour, des tonalités sombres et cendrées devant le mur de fond à la transparence à peine colorée près de la manche du personnage qui relève un pan de son voile. Cette nouvelle technique picturale permet d'aborder aussi avec précision certains détails techniques du tissu drapé : ainsi dans un portrait officiel de Marguerite d'York, épouse de Charles le Téméraire depuis 1468 (fig. 4). Son long voile transparent est plissé en panneaux horizontaux, dits « en vitres » - effet obtenu en pliant l'étoffe et en appuyant fortement sur le pli avec un fer. Il s'agit là d'un effet de transparence que peintres et enlumineurs ont souvent reproduit mais dont les jeux de réfraction de lumière sont particulièrement saisis par la technique de la peinture à l'huile.

\section{Entre nudité et transparence}

\section{- entre pureté et transgression}

Dans un autre registre, la déshérence du corps condamné (les corps torturés du Jugement Dernier) a longtemps été figurée par une nudité que même le tissu transparent ne saurait mitiger. À la fin du Moyen Âge, au XV siècle surtout, cette thématique s'accroît de certains récits antiques et mythologiques qui autorisent le déploiement complet de la nudité, par exemple le récit d'Agrippine disséquée sous les ordres de Néron, dans le Roman de la Rose (Guillaume de Lorris, 1230-1235 et Jean de Meung, 1275-1280), et ses illustrations. La nudité partielle du corps exposé par un vêtement déchiré et troué, rendu quasitransparent, signe de déchéance et d'exclusion, prête à un jeu littéraire suggestif autour des femmes victimes, Griseldis ${ }^{35}$ ou Énide ${ }^{36}$, ou incite au rejet catégorique de Pauvreté dans le Roman de la Rose. Dans l'illustration manuscrite abondent les scènes à la maison de bains, où hommes et femmes sont nus mais conservent leur habillement de tête pour consommer repas et rendez-vous amoureux. Apparaissent alors des représentations plus rares où la nudité est présentée de face, et à peine cachée par un voile transparent que la femme fait délicatement flotter autour d'elle sans couvrir autre

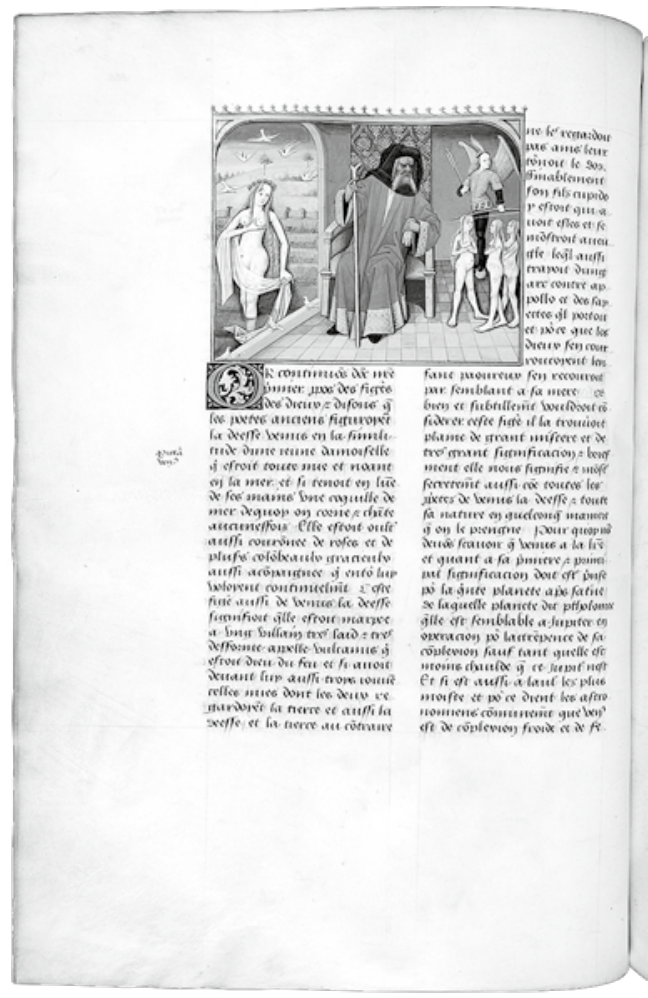

5. Evrard de Conty, Robinet Testard, Le livre des Échecs Amoureux moralisé, 1401 1500, Paris, BnF, département des Manuscrits, Fr. 143 , fol. $104 v^{\circ}$.

chose que son sexe. Séduction, péché, menace, sorcellerie, sont autant de dimensions présentes dans le célèbre tableau de la sorcière au " philtre d'amour " (Der Liebeszauber) peint par un maître anonyme allemand ${ }^{37}$ et dans une Vénus au bain aperçue à travers une croisée, peinte par Robinet Testard $^{38}$ (fig. 5).

L'extrême finesse des tissus dépeints dans les arts visuels de la fin du Moyen Âge a pu amener une spécialiste de l'histoire du vêtement, Maria Giuseppina Muzarelli, à se demander s'ils étaient effectivement reproduits ou bien imaginés pour exercer l'art des peintres ${ }^{39}$. Dans l'étude des tissus transparents, le contraste frappe en effet entre la fervente expression de la luminosité que leur ont imprimée les objets picturaux et la sècheresse des attestations matérielles. Manuscrits illustrés et tableaux de la fin du Moyen Âge ont souligné l'impact technique et esthétique de ces textiles mais sans en éclipser ni les paradoxes ni la valeur performative. Ces tissus et les effets de transparence qu'ils produisent sont-ils un simple effet de mode, ou une attestation de luxe économique, ou bien encore l'un des éléments d'un cérémonial courtois façonné par les élites ? Sont-ils représentés dans les images profanes 
en miroir de leur présence dans l'iconographie sacrée, ou comme contrepoids symbolique au déploiement fastueux de joyaux et d'étoffes luxueuses? Les tissus diaphanes du Moyen Âge, attestés ou représentés, ne laissent donc pas de susciter notre interrogation. Ainsi, des notions telles que le secret, la pureté, l'interdit d'une part, et la consommation et le pouvoir de l'autre, peuvent enrichir considérablement les sens culturels rattachés aux tissus transparents à la fin du Moyen Âge. Si leur fabrication, leur diffusion, et leur valeur d'échange exigent une recherche plus poussée sur la base des documents commerciaux, juridiques et techniques, il nous paraît tout aussi indispensable de poursuivre une analyse plus étendue des signes visuels que la transparence textile suscite en créant des espaces d'intersection entre sacré et profane.

Francesca Canadé Sautman, Hunter College

et Graduate Center of the City University of New York FSautman@gc.cuny.edu 
1. British Library, Add MS 42130, fol. $76 \mathrm{v}^{\circ}$, bas de page, centre. Autres exemples de dames aux pans de voile envolés dans ce manuscrit : folios $35 \mathrm{r}^{\circ}, 61 \mathrm{r}^{\circ}, 75 \mathrm{v}^{\circ}$. On trouve ce même motif dans d'autres manuscrits du XIVe siècle, comme le Ormesby Psalter (Oxford, Bodleian Library, MS Douce 366) ou le Roman d'Alexandre (Oxford, Bodleian Library, MS Bodley 264); il semble signaler le trouble érotique et faire écho au thème des "Vierges folles".

2. L'érudition ne semble pas s'être penchée sur cette typologie textile en tant que telle, que ce soit dans l'important article de Françoise Piponnier, " À Propos des textiles anciens, principalement médiévaux ", dans Annales. Economies, Sociétés, Civilisations, $22^{\mathrm{e}}$ année, 4, 1967, p. 864-880, ou dans l'ouvrage recent de Bart Lambert et Katherine Anne Wilson (dir.), Europe's Rich Fabric: The Consumption, Commercialization and Production of Luxury Textiles in Italy, the Low Countries and Neighbouring Territories (Fourteenth-Sixteenth Centuries), Farnham [u.a.], 2015.

3. Camille Enlart, Manuel d'archéologie française depuis les temps mérovingiens jusqu'à la Renaissance, III. Le Costume, Paris, 1916, p. 20-22; pour les coiffures des XIV et $\mathrm{XV}^{\mathrm{e}}$ siècles, p. 186-213.

4. Utilisant un métier à main, la technique du sprang permettait d'obtenir un produit élastique en forme de filet résultant de la torsion de fils de trame parallèles, connu en Grèce antique et sous la colonisation romaine. John Peter Wild, Penelope Walton Rogers, "Introduction", dans David T. Jenkins (dir.), The Cambridge History of Western Textiles, Cambridge [u.a.], 2003, p. 25 ; Ian Jenkins, "The Greeks ", dans David T. Jenkins (dir.), op. cit., p. 75, et John Peter Wild, "The Romans in the West, 600 BC - AD 400 ", dans David T. Jenkins (dir.), op. cit., p. 88 .

5. James Robinson Planché, "Crispine ", dans A Cyclopaedia of Costume, or Dictionary of Dress including Notices of Contemporaneous Fashions..., 2 vol., Londres, 1876, I, p. 147.

6. Penelope Walton Rogers, "Anglo-Saxons and Vikings in Britain, AD $450-1050$ », dans Jenkins, 2003, cité n. 4, p. 131-132.

7. Victor Gay, Glossaire archéologique du Moyen Âge et de la Renaissance, Paris, 1887, I, p. 494. Gay mentionne une bible conservée à Bologne mais les bibles de Théodulfe sont cataloguées au nombre de trois : Bibles dite de SaintHubert (Londres, The British Museum, Add. 24142), du Puy (trésor de la cathédrale) et d'Orléans (Paris, BnF, lat. 9380). Il s'agit vraisemblablement de celle du Puy, objet d'une notice de Philippe Hedde, Essai paléographique sur un manuscrit enrichi de tissus du IX' siècle, au Puy, 1839, cité dans Léopold Delisle, "Les Bibles de Théodulfe", dans Bibliothèque de l'École des Chartes, 40, 1, 1879, p. 5-47, note 2 .

8. Gay, 1887, cité n. 7, p. 493-494.

9. Agnes Geijer, A History of Textile Art, Londres, 1979, p. 57.

10. Robert S. Lopez, "Nouveaux documents sur les marchands italiens en Chine à l'époque mongole ", dans Comptes rendus des séances de l'Académie des Inscriptions et Belles lettres, 121, 2, 1977, p. 445-458.
11. Laurence Gérard-Marchant, "Compter et nommer l'étoffe à Florence au Trecento (1343) ", dans Michel Pastoureau (dir.), "L'étoffe et le vêtement ", numéro thématique de Médiévales, 14, 29, 1995, p. 87-104.

12. Sur les lois somptuaires et leurs études anciennes : Sarah-Grace Heller, "Limiting Yardage and Changes of Clothes: Sumptuary Legislation in Thirteenth-Century France, Languedoc, and Italy ", dans E. Jane Burns (dir.), Medieval Fabrications: Dress, Textiles, Clothworks, and Other Cultural Imaginings, New York [u.a.], 2004, p. 121-136.

13. Voir l'article "Gazzatum ", dans Planché, 1876, cité n. 5, p. 202, qui mentionne l'interdiction de porter de la gaze et " toute autre forme de tissu particulièrement fin " ( « et allium quemcunque pannum notabiliter delicatum, interdicimus universis "), et l'article "Nifels ", dans Planché, 1876, cité n. 5, p. 379.

14. Enlart, 1916, cité n. 3, p. 54 : «bande de toile ou de couvrechef, c'est-à-dire du linon qui servait à faire les voiles de tête" ".

15. Enlart, 1916, cité n. 3, p. 11-12; et p. 213-216: " atours de lingerie ". Léon de Laborde, "Atours ", dans Glossaire français du Moyen Âge à l'usage de l'archéologue et de l'amateur des arts..., Paris, 1872, ad vocem.

16. Kurt Zangger, Contribution à la terminologie des tissus en ancien français attestés dans les textes français, provençaux, italiens, espagnols, allemands et latins, Bienne, 1945, p. 9-15.

17 Eunice Rathbone Goddard, Women's Costume in French Texts of the Eleventh and Twelfth Centuries, Baltimore/Paris, 1927 ; cet ouvrage donne déjà des indications lexicales bien trop nombreuses pour être reproduites ici, voir " chainse délié ", p. 70; " chemise ", p. 91-97, " chemises déliées ", avec cinq exemples, p. $92-93$; " guimpe déliée ", p. 138 ; « lier », p. 151-153.

18. Goddard, 1927, cité n. 17, p. 77, et « chensis blancs, deliez ", p. 76.

19. Goddard, 1927, cité n. 17, p. 92 : «plus fine que fil d'araignée ".

20. Jean-Guy Goutebroze, "Entre le nu et le vêtu : le transparent ", dans Le nu et le vêtu au Moyen Âge: XII XIII siècles, actes de colloque (Aix-en-Provence, 2000), Aix-en-Provence, 2001, p. 113-121.

21. Voir l'excellent essai de Romaine Wolf-Bonvin, "La chemise : un vêtement sans l'être ", dans Le nu et le vêtu..., 2001, cité n. 20, p. 383-394.

22. Alfred Franklin, "Atourneresses", dans Dictionnaire historique des arts, métiers et professions exercés dans Paris depuis le treizième siècle, Paris/Leipzig, 1906, p. 52.

23. Gay, 1887, cité n. 7, p. 485.

24. Goddard, 1927, cité n. 17, p. 221-222.

25. Nombre de peintres ont peint la Crucifixion ou la Descente de Croix avec le pagne transparent durant ou à la fin du XIV e siècle, utilisant la technique de la tempera sur bois, dont : Anonyme, Triptyque, 1333, musée du Louvre, inv. 20197 ; Bernardo Daddi, Triptyque de la Crucifixion, $2^{\mathrm{e}}$ quart du XIV ${ }^{\mathrm{e}}$ siècle, musée du Louvre ; Simone Martini, Descente de Croix, 1333, Anvers, 
Koninklijk Museum voor Schone Kunsten ; Maître anonyme de Norwich, Retable de la Passion, vers 1380 1390, cathédrale de Norwich, chapelle de Saint-Luc ; Jean de Baumetz, Crucifixion avec un moine cartusien en prière, 1389, musée du Louvre ; Pedro Serra, Retable de la Vierge avec l'Enfant et les saints, 1394, Manresa, chapelle du Saint Esprit ; Jean Malouel, La Grande Pietà Ronde, vers 1400, musée du Louvre ; Konrad von Soest, Mont du Calvaire, panneau central du Retable la Passion, 1403 , Bad Wildungen (Allemagne), église paroissiale.

26. Crucifixion, Staatliche Museen zu Berlin.

27. Jean-Claude Schmitt, Jérôme Baschet, "La "sexualité" du Christ ", dans Annales. Economies, Sociétés, Civilisations, 46, 2, 1991, p. 337-346.

28. Jennifer P. Kingsley, " Picturing the Treasury: The Power of Objects and the Art of Memory in the Bernward Gospels ", dans Gesta, 50, 1, 2011, p. 19-39; p. 26.

29. Atelier de Botticelli, Vierge à l'Enfant avec saint Jean et un ange, tempera sur bois, vers 1490, Londres, National Gallery.

30. Giovanni Boccaccio, Robinet Testard (enluminure), Des cleres et nobles femmes, trad. fra. anonyme de De Claris mulieribus, vers 1488-1496, Paris, BnF, département des Manuscrits, Ms. Français 599, fol. 54r $\mathrm{r}^{\circ}$.

31. Soulignons que ces drapés diaphanes appartiennent à une aire très précise, le domaine Bourguignon-Flamand, la moitié Nord de la France, l'Angleterre, une partie des régions germaniques, mais ne sont pas usités en Castille par exemple ou dans les pays slaves.

32. Diane Wolfthal, The Beginnings of Netherlandish Canvas Painting: 1400-1530, New York/Cambridge, 1989, p. 26-27.

33. Pièce de lin plissé portée au-dessous ou au-dessus du menton, la barbe ou barbette blanche est un signe de deuil noble. Planché, 1876, cité n. 5, p. 34. Sur Marguerite d'Autriche et sa performance de la viduité, voir Women of Distinction: Margaret of York, Margaret of Austria, Dagmar Eichberger (dir.), cat. exp. (Mechelen, Lamot, 2005), Louvain, 2005.

34. Sur ce tableau, voir Lorne Campbell, Yvonne Szafran, "The Portrait of Isabella of Portugal, Duchess of Burgundy, in the J. Paul Getty Museum ", dans The Burlington Magazine, 146, 1212, March 2004, p. 148-157.

35. Roberta L. Krueger, "Uncovering Griselda: Christine de Pizan, "une seule chemise", and the Clerical Tradition: Boccaccio, Petrarch, Philippe de Mézières, and the Ménagier de Paris", dans Burns, 2004, cité n. 12, p. 71-88.

36. Voir Goutebroze, 2001, cité n. 20, sur Érec et Énide de Chrétien de Troyes.

37. Bas-Rhin, Anonyme, Der Liebeszauber, $2^{\mathrm{e}}$ moitié du XVe siècle, Leipzig, Museum der bildenden Künste.

38. Evrart de Conty, Le livre des échecs amoureux moralisés illustrés par Robinet Testard, Paris, BnF, département des Manuscrits, ms. Fr. 143, fol. $104 v^{\circ}$. Un autre document des plus curieux est la Bible du Roi Wenceslas de Bohème, fin $\mathrm{XIV}^{\mathrm{e}}$ siècle - début $\mathrm{XV}^{\mathrm{e}}$ siècle, ornée dans les marges de figures de " filles de bains " portant brosse et combinaison légère dont au moins une est entièrement transparente et découvre une nudité frontale complète (Vienne, Österreichischen Nationalbibliothek, Codices vindobonenses 2759-2764).

39. Maria Giuseppina Muzzarelli, "Statuts et identités. Les couvre-chefs féminins (Italie centrale, $\mathrm{XV}^{\mathrm{e}}-\mathrm{XVI}^{\mathrm{e}}$ siècle) ", dans Clio, Femmes, Genre, Histoire, 36, 2012 , p. 67-89, en ligne : http://clio.revues.org/10748, consulté le $12 / 05 / 2016$. 